\title{
Clinical, Pathological, Treatment Pattern and Post-management Follow-up Features of Differentiated Thyroid Carcinoma (DTC) in a Tertiary Level Health Care Center of Bangladesh
}

\author{
Parvez Ahmed*1, Subrata Ghosh ${ }^{2}$, Mostafa Shamim Ahsan ${ }^{3}$, Nasrin Begum ${ }^{4}$, \\ Mosharruf Hossain ${ }^{5}$, M.M. Arif Hosen ${ }^{6}$
}

\begin{abstract}
Introduction: This study evaluates clinical, pathological, treatment pattern and post-management follow-up features of differentiated thyroid carcinoma affected patients who had attended Rajshahi Medical College Hospital as well as private clinics and then Institute of Nuclear Medicine \& Allied Sciences, Rajshahi for primary surgical management and post-operative radioiodine ablation therapy respectively. Materials and Methods: It is a retrospective study. Clinico-pathological, treatment and post-management follow-up features of 254 patients of histologically proved differentiated thyroid carcinoma (DTC) were recorded from their ultrasonography report, pre-operative cytology, operation note, post-operative histopathology and radioiodine ablation therapy related follow-up book between 2011 and 2015 and analyzed using statistical software IBM SPSS v. 16. Results: Among the sample $(n=254), 211(83 \%)$ were female and 43 (17\%) were male. Mean age group was 30-39 years. In relation to FNAC findings of thyroid nodules among this study sample $(n=254), 223$ (88\%) had malignant cytology, $16(6 \%)$ had borderline cytology and 15 (6\%) had benign cytology. Regarding the histopathological findings of thyroid nodules, 241 (95\%) had papillary carcinoma and 13 (5\%) had follicular carcinoma. Regarding radioiodine ablation dose, 198 (78\%) had been given 138 millicurie (5.1 gigabecquerel) and 56 $(22 \%)$ had been given 178 millicurie (6.6 gigabecquerel). Conclusion: Early and successful management of differentiated thyroid carcinoma is required to practice widely and equitably in order to significantly reduce mortality and morbidity related to such conditions.
\end{abstract}

Keywords: Differentiated thyroid carcinoma (DTC), Clinical features, Pathological features, Treatment pattern, Postmanagement follow-up features.

Number of Figures: 06; Number of References: 12; Number of Correspondences: 03.

*1. Corresponding Author:

\section{Dr. Parvez Ahmed}

Assistant Professor

Institute of Nuclear Medicine \& Allied Sciences

Rajshahi, Bangladesh.

Email: drparvezahmed@yahoo.com

2. Dr. Subrata Ghosh

Assistant Professor

Department of Ear, Nose, Throat \& Head Neck Surgery

Rajshahi Medical College Hospital, Rajshahi.

3. Prof. Dr. Mostafa Shamim Ahsan

Director

Institute of Nuclear Medicine \& Allied Sciences

Rajshahi, Bangladesh.

4. Dr. Nasrin Begum

Associate Professor

Institute of Nuclear Medicine \& Allied Sciences Rajshahi, Bangladesh.

5. Dr. Mosharruf Hossain

Associate Professor

Institute of Nuclear Medicine \& Allied Sciences Rajshahi, Bangladesh.

6. Dr. M.M. Arif Hosen

Assistant Professor

Institute of Nuclear Medicine \& Allied Sciences

Rajshahi, Bangladesh.

\section{Introduction:}

Well-differentiated thyroid carcinoma that arises from the thyroid follicular epithelial cells is the most common endocrine neoplasia ${ }^{1,2}$. Among the well-differentiated thyroid carcinomas, the incidence of papillary thyroid carcinoma rose by a factor of three around the world from 1973 to 2009 and most of the increased incidence is traceable to more frequent diagnostic evaluation with improved technologynamely, more widespread and aggressive use of ultrasound and image-guided biopsy ${ }^{3,4,5,6}$. Regarding treatment, well-differentiated carcinomas of the thyroid are treated by surgery combined with radioactive iodine therapy ${ }^{7}$. In fact, various surgical techniques have been devised for tumor invasion confined to the neck depending on the site and longitudinal and transverse extent of tumor ${ }^{8}$.

\section{Materials and Methods:}

It is a retrospective study. Clinico-pathological, treatment and post-management follow-up features of 254 patients of histologically proved differentiated thyroid carcinoma (DTC) who had attended Rajshahi Medical College Hospital and then Institute of Nuclear Medicine \& Allied Sciences, Rajshahi for primary surgical management and post-operative radioiodine ablation therapy respectively were recorded from their ultrasonography report, pre-operative cytology, operation note, post-operative histopathology and radioiodine ablation therapy related follow-up book between 2011 and 2015. Their data were analyzed using statistical software IBM SPSS v. 16.

Results:

Among the sample $(\mathrm{n}=254), 211(83 \%)$ were female and $43(17 \%)$ were male (Figure-1). 


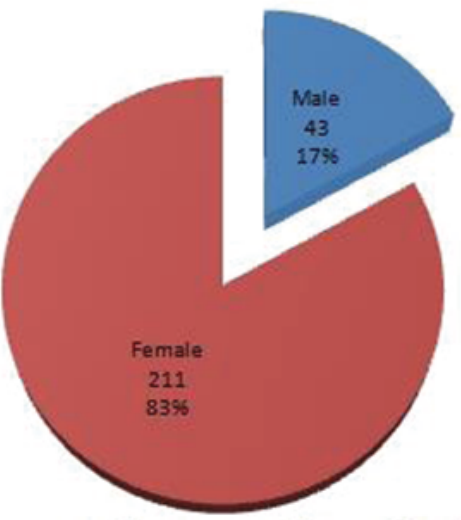

Figure-1: Distribution of gender $(\mathrm{n}=254)$.

Mean age group was 30-39 years (Figure-2).

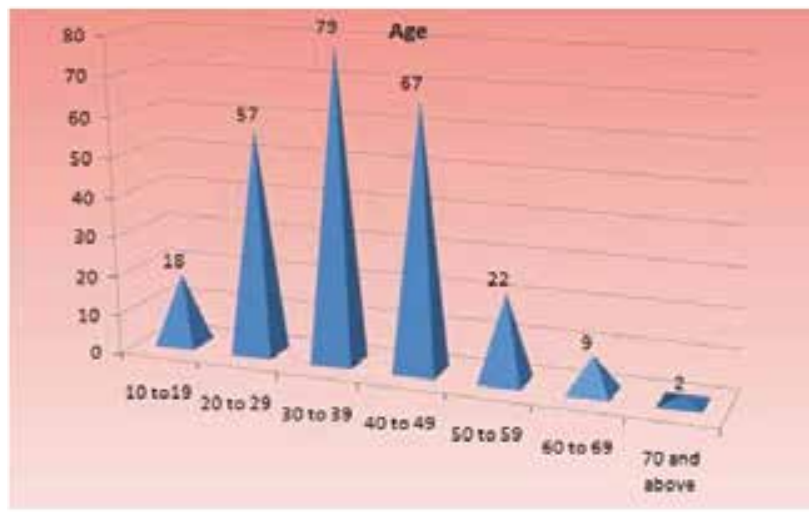

Figure-2: Distribution of age $(n=254)$.

Regarding the USG findings of thyroid gland, 187 (74 \%) had unilateral nodule and 67 (24\%) had bilateral nodules (Figure-3).

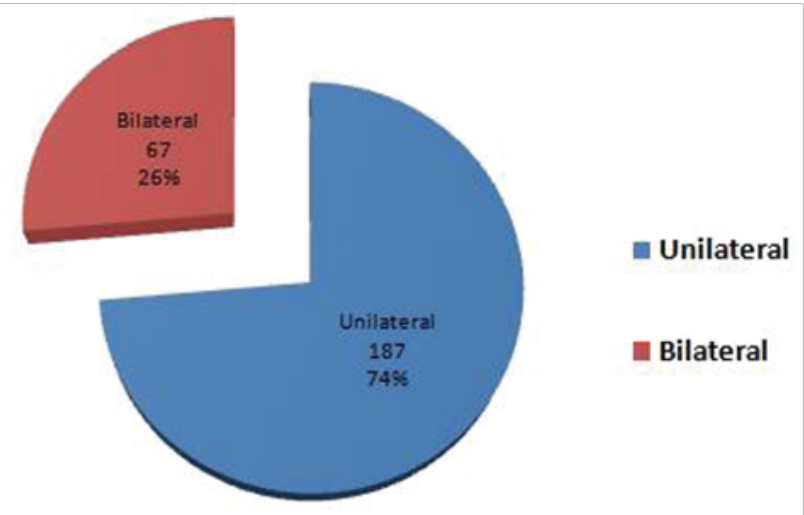

Figure-3: HRUS findings of thyroid nodules $(\mathrm{n}=254)$.

And, in relation to FNAC findings of thyroid nodules among this study sample $(\mathrm{n}=254), 223 \quad(88 \%)$ had malignant cytology, $16(6 \%)$ had borderline cytology and $15(6 \%)$ had benign cytology (Figure-4).

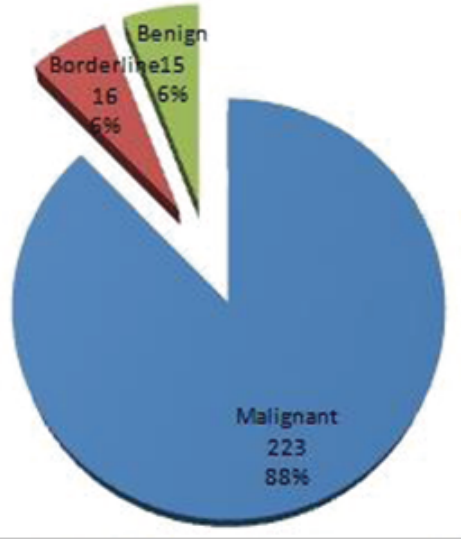

E Malignant

E Borderline

E Benign

Regarding the histopathological findings of thyroid nodules, 241 (95\%) had papillary carcinoma and $13(5 \%)$ had follicular carcinoma (Figure-5).

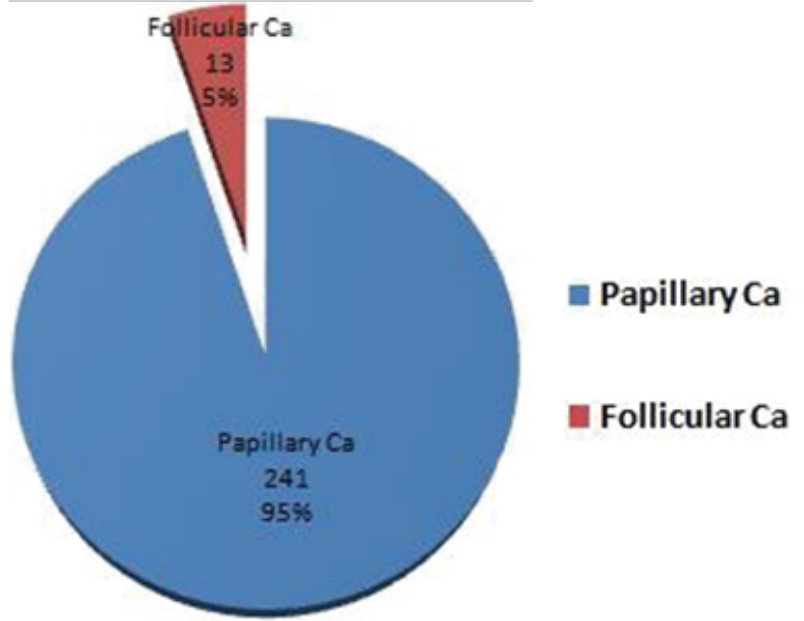

Figure-5: Histopathology findings of thyroid nodules $(n=254)$.

In relation to surgical management, 185 (73\%) had undergone total thyroidectomy with central clearance, 48 $(19 \%)$ had undergone total thyroidectomy with selective neck dissection, $13(5 \%)$ had undergone completion thyroidectomy and 8 (3\%) had undergone total thyroidectomy with modified radical neck dissection (Figure-6).

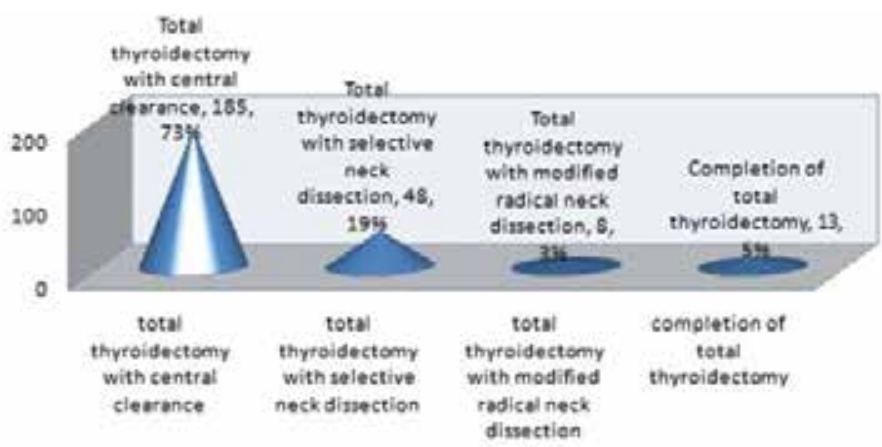

Figure-6: Types of undertaken neck surgery $(n=254)$. 
Regarding radioiodine ablation dose, $198(78 \%)$ had been given 138 millicurie (5.1 gigabecquerel) and $56(22 \%)$ had been given 178 millicurie (6.6 gigabecquerel) And, in relation to treatment outcome from 2011 to 2016 among the sample $(n=254), 26(10 \%)$ were treated in 2011 and had recurred once (in 2014), 49 (20\%) were treated in 2012 and had recurred twice (once in 2015 and once in 2016), 46 $(18 \%)$ were treated in 2013 and had recurred twice in 2016, $49(19 \%)$ were treated in 2014 and had recurred once in 2016 and $84(33 \%)$ were treated in 2015 and had no recurrence upto 2016.

\section{Discussion:}

Thyroid cancer is the most common endocrine malignancy. The most frequent histologic subtype of thyroid carcinoma is papillary (accounting for $80.2 \%$ of cases), followed by follicular carcinoma ( $11.4 \%$ of cases $)$, which are commonly collectively referred to as well-differentiated thyroid cancer ${ }^{9}$. In the systematic investigation of thyroid nodules, risk stratification is performed on the basis of the physical, ultrasonographic, and scintigraphic findings, and the diagnosis is generally established by fine-needle biopsy and cytology ${ }^{10}$.

Papillary thyroid microcarcinoma (PTMC) is a specific subgroup of papillary thyroid carcinoma (PTC) and defined by WHO on the largest dimension of $1.0 \mathrm{~cm}$ or less. Most of PTMC are not detectable at clinical examination and are diagnosed incidentally during pathologic examination of thyroid specimens after surgery for benign thyroid diseases, or in autopsies. Characteristic cytologic features of PTC help make the diagnosis by FNA or after surgical resection; these include psammoma bodies, cleaved nuclei with an "orphan-Annie" appearance caused by large nucleoli, and the formation of papillary structures. Moreover, the increased accuracy of the pathologic thyroid examination, in particular with the thinness and the number of the anatomical slices obtained for thyroid specimens, led to a more frequent pathologic; diagnosis of incidental PTMC. On the other hand, the widespread use and the technical improvement of thyroid ultrasonography and fine-needle aspiration biopsy (FNAB) contributed to an increase in the rate of preoperative diagnosis of PTMC over the last few decades ${ }^{11}$.

\section{Conclusion:}

The incidence of thyroid cancer has increased dramatically during the past three decades and it is now the fastest growing cancer in women ${ }^{12}$. Therefore, early and successful management of differentiated thyroid carcinoma is required to practice widely and equitably in order to significantly reduce mortality and morbidity related to such conditions. Through the findings of this study, such well-established management protocol has been highlighted at this tertiary level at Rajshahi, Bangladesh. However, large-scale relevant studies are required to evaluate ongoing management protocol so that global standard can be achieved.

Conflict of Interest: None.

\section{Acknowledgement:}

I would like to thank all the co-authors especially Dr. M. M. Arif Hosen who have encouraged and helped me a lot in preparing this article. I would also like to thank Mr. Md. Masudur Rahman, Nuclear Medicine Technologist of INMAS, Rajshahi.

\section{References:}

1.Ceresini G, Corcione L, Michiara M. Thyroid cancer incidence by histological type and related variants in a mildly iodine-deficient area of Northern Italy, 1998 to 2009. Cancer. 2012; 118: 5473-5480. [PubMed]

https://www.ncbi.nlm.nih.gov/pubmed/22517468 DOI:10.1002/cncr.27591

2. Robert Koch-Institut und Gesellschaft der epidemiologischen Krebsregister in Deutschland e. V. (eds.): Krebs in Deutschland 2009/2010. 9. Ausgabe. Berlin, 2013.

https://www.aerzteblatt.de/int/archive/references?id=171082

DOI: $10.3238 /$ arztebl.2015.0452

3. Morris LGT, Sikora AG, Tosteson TD, Davies L. The increasing incidence of thyroid cancer: The influence of access to care. Thyroid. 2013; 23: 885-891. [PMC free article] [PubMed]

https://www.ncbi.nlm.nih.gov/pubmed/23517343

DOI: $10.1089 /$ thy. 2013.0045

4. Tuttle RM, Ball DW, Byrd D. Thyroid carcinoma. J Natl Compr Canc Netw. 2010; 8: 1228-1274. [PubMed]

https://www.ncbi.nlm.nih.gov/pubmed/21081783

PMID: 25505208

5. Davies L, Welch HG. Increasing incidence of thyroid cancer in the United States, 1973-2002. JAMA. 2006; 295 : 2164-2167.

https://www.ncbi.nlm.nih.gov/pubmed/16684987

DOI: $10.1001 /$ jama.295.18.2164

6. Cooper DS, Doherty GM, Haugen BR. Management guidelines for patients with thyroid nodules and differentiated thyroid cancer. Thyroid. $2006 ; 16: 109-142$.

https://www.ncbi.nlm.nih.gov/pubmed/16420177

DOI: $10.1089 /$ thy. 2006.16 .109

7. Dralle H, Musholt TJ, Schabram J, et al. German Association of Endocrine Surgeons practice guideline for the surgical management of malignant thyroid tumors. Langenbecks Arch Surg. 2013;398:347-375. [PubMed]

https://www.ncbi.nlm.nih.gov/pubmed/23456424

DOI: $10.1007 / \mathrm{s} 00423-013-1057-6$

8. Lorenz K, Niederle B, Steinmüller T, Dralle H. The European Society of Endocrine Surgeons perspective of thyroid cancer surgery: an evidence-based approach. Langenbecks Arch Surg. 2014; 399:135-139. [PubMed] https://www.ncbi.nlm.nih.gov/pmc/articles/PMC4515787/ DOI: 10.3238/arztebl.2015.0452 
9. Annual Report to the Nation on the status of cancer, 1973-1999, featuring implications of age and aging on U.S. cancer burden

https://onlinelibrary.wiley.com/doi/full/10.1002/cncr.10593? sid=nlm\%3Apubmed

DOI: $10.1002 /$ cncr. 10593

10. American Association of Clinical Endocrinologists, Associazione Medici Endocrinologi, and European Thyroid Association Medical guidelines for clini... - PubMed - NCBI https://www.ncbi.nlm.nih.gov/pubmed/20551008

DOI: $10.4158 /$ EP. 16.3 .468
11. Papillary thyroid microcarcinoma: extrathyroidal extension, lymph node metastases, and risk factors for recurrence in a high prevalence of goiter a... - PubMed - NCBI https://www.ncbi.nlm.nih.gov/pubmed/20052467 DOI: $10.1007 / \mathrm{s} 00268-009-0375-\mathrm{X}$

12. Bilimoria KY, Bentrem DJ, Ko CY. Extent of surgery affects survival for papillary thyroid cancer. Ann Surg. 2007; 246 :375-381. [PMC free article] [PubMed] https://www.ncbi.nlm.nih.gov/pubmed/17717441

DOI: $10.1097 /$ SLA.0b013e31814697d9 